\title{
Fabry-Pérot Cavity Based on a Suspended-Core Fiber for Strain and Temperature Measurement
}

\author{
Orlando Frazão, S. H. Aref, José M. Baptista, José L. Santos, H. Latifi, F. Farahi, Jens Kobelke, and Kay Schuster
}

\begin{abstract}
A fiber-optic Fabry-Pérot sensing structure based on the utilization of a suspended-core fiber is presented. The interferometric structure is formed when a small length of the suspendedcore fiber is spliced to the end of a standard single-mode fiber. The interfering waves are generated by the refractive-index mismatches between the two fibers in the splice region and at the end of the suspended-core fiber. Thermal and strain responses of two different sensing heads associated with suspended-core fibers with three and four holes are characterized.
\end{abstract}

Index Terms-Fabry-Pérot (FP) interferometer, microstructured fiber, optical fiber sensor.

\section{INTRODUCTION}

$\mathbf{O}$ PTICAL fiber Fabry-Pérot (FP) interferometric sensors have been demonstrated in the past for many sensing applications. These sensors can be classified as extrinsic or intrinsic depending on their geometry. For extrinsic sensors, an air gap between two cleaved end faces is used. On the other hand, when dealing with intrinsic sensors, the mirrors are fabricated inside the fiber. Kersey et al. [1] published in 1983 a fiber FP formed by the two extremes of the fiber. In 1988, Lee and Taylor [2] proposed a fiber FP where the FP reflectors were formed on the single-mode fiber by a fusion splicing technique. Farahi et al. [3] demonstrated a multiplexing network using this type of

Manuscript received April 07, 2009; revised May 15, 2009. First published July 10, 2009; current version published August 19, 2009. This work was performed in the framework of European Program COST, Action 299 "Optical Fibers for New Challenges Facing the Information Society.'

O. Frazão is with the Instituto de Engenharia de Sistemas e Computadores do Porto (INESC Porto), 4169-007 Porto, Portugal (e-mail: ofrazao@ inescporto. pt).

S. H. Aref is with the Instituto de Engenharia de Sistemas e Computadores do Porto (INESC Porto), 4169-007 Porto, Portugal, and also with the Laser and Plasma Research Institute, Shahid Beheshti University, Tehran 1983963113, Iran.

J. M. Baptista is with the Instituto de Engenharia de Sistemas e Computadores do Porto (INESC Porto), 4169-007 Porto, Portugal, and also with the Departamento de Matematica e Engenharias, Universidade da Madeira, 9000-390 Funchal, Portugal (e-mail: jmb@inescporto.pt).

J. L. Santos is with the Instituto de Engenharia de Sistemas e Computadores do Porto (INESC Porto), 4169-007 Porto, Portugal, and also with the Departamento de Física, Faculdade de Ciências, Universidade do Porto, 4169-007 Porto, Portugal (e-mail: josantos@fc.up.pt).

H. Latifi is with the Laser and Plasma Research Institute, Shahid Beheshti University, Tehran 1983963113, Iran.

F. Farahi is with the Department of Physics, University of North Carolina at Charlotte, Charlotte, NC 28223 USA.

J. Kobelke and K. Schuster are with the Institute of Photonic Technology (IPHT), D-07745 Jena, Germany (e-mail: jens.kobelke@ipht-jena.de; kay.schuster@ipht-jena.de).

Color versions of one or more of the figures in this letter are available online at http://ieeexplore.ieee.org.

Digital Object Identifier 10.1109/LPT.2009.2024645 optical fiber sensors. Kao and Taylor [4] reported the same configuration located inside of a stainless-steel hypotube to act as a pressure sensor. Later, Tsai and Lin [5] demonstrated an intrinsic FP temperature sensor by fusing two fibers with different core diameters.

Recently, with the emergence of the photonic crystal fibers (PCFs) [6], several authors have studied and also used this new technology to implement new devices, namely novel FP-fiber-based interferometers [7]-[9]. An FP structure formed by splicing a section of hollow-core PCF between two single-mode fibers was proposed and demonstrated by Rao et al. [10], where a reflective $\mathrm{Ti}_{2} \mathrm{O}_{3}$ film was coated on the end surface of the lead-out fiber to form a Fizeau-type etalon. This sensing device shows good sensitivity to strain and low sensitivity to temperature. A miniature in-line fiber-optic FP etalon, micromachined by a femtosecond $157-\mathrm{nm}$ laser, was fabricated on a PCF and reported by Ran et al. [11]. Due to the low coefficient of thermal expansion of silica and the residual thermooptic coefficient of air, it was reported nearly temperature independent strain measurement up to $800{ }^{\circ} \mathrm{C}$. The suspended-core fiber structure was first proposed by Monro et $a l$. [12] and one of the initial envisaged applications was gas sensing. Indeed, Webb et al. [13] investigated an acetylene gas sensor based on a suspended-core fiber. Recently, Frazão et al. [14] characterized strain and temperature response of a Sagnac interferometer sensor with a four-hole suspended-core fiber. In this letter, a new geometry of an FP structure based on a suspended-core fiber with three and four holes is presented. The sensors' characteristics for strain and temperature measurements are reported.

\section{EXPERIMENTAL RESULTS}

Fig. 1 presents the experimental setup for characterizing the sensing head. The setup consists in a broadband source with a bandwidth of $100 \mathrm{~nm}$ and a central wavelength of $1550 \mathrm{~nm}$. The FP was interrogated in reflection via an optical circulator and an Advantest Q8384 Optical Spectrum Analyzer (OSA), with a maximum resolution of $10 \mathrm{pm}$.

The FP cavity is formed when a small section of a suspendedcore fiber (with three or four holes) is spliced at the end of a standard single-mode fiber (SMF 28). The loss induced by the splice is $\approx 5 \mathrm{~dB}$. The suspended-core fibers were made of pure silica and fabricated at Institute of Photonic Technology (IPHT, Jena, Germany). One of the fibers has three holes with diameters of $\approx 20 \mu \mathrm{m}$, while the core and the cladding have diameters of $\approx 3.2$ and $\approx 129 \mu \mathrm{m}$, respectively. The other suspended-core fiber used has four holes with diameters of $\approx 43 \mu \mathrm{m}$, a core of $\approx 5 \mu \mathrm{m}$, and a cladding with $\approx 139 \mu \mathrm{m}$ (inset of Fig. 1). The 


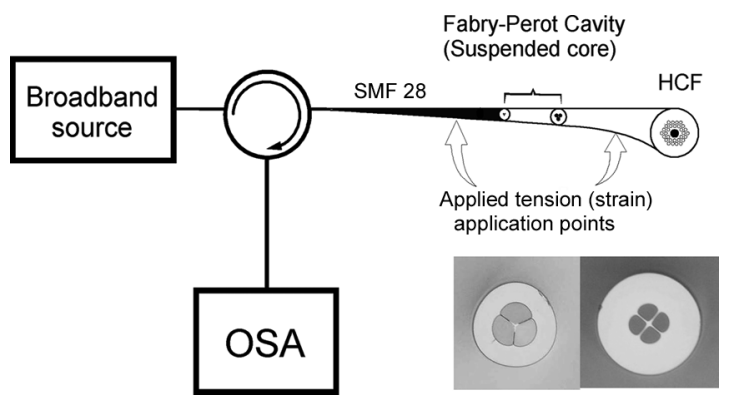

Fig. 1. Experimental setup with the sensing head (photographs of the suspended-core fiber with three and four holes are shown).

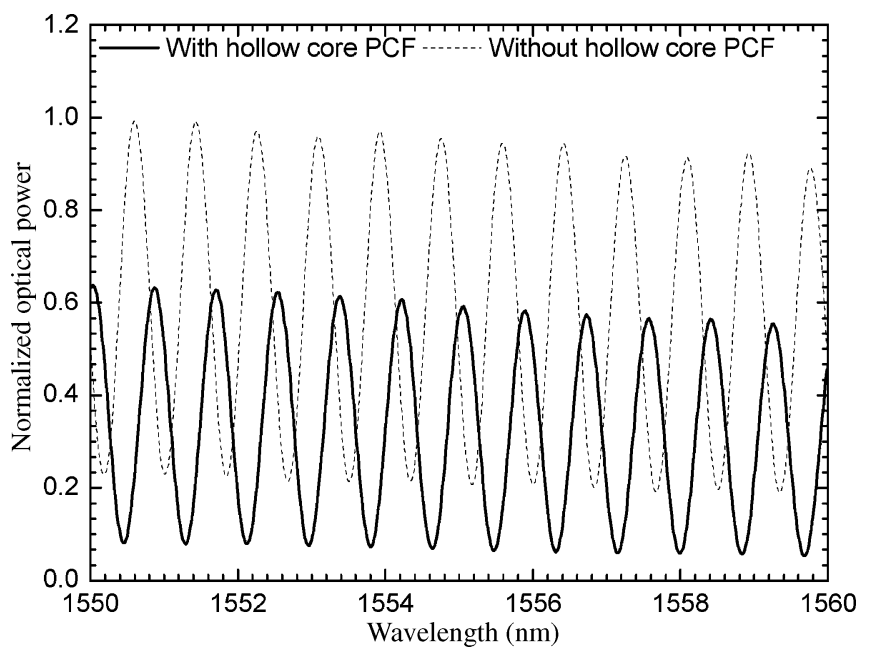

Fig. 2. Spectral response of the FP cavity with a four-hole suspended-core fiber before and after the splicing with a hollow-core fiber.

two suspended-core fibers under test had a length $\approx 1 \mathrm{~mm}$ (four holes) and $\approx 0.84 \mathrm{~mm}$ (three holes). Due to this small length and in order to be able to apply strain, a hollow-core fiber (in this case, a hollow-core PCF) with $50 \mathrm{~mm}$ length was spliced at the end of the suspended-core fiber without destroying the core mirrored surface of this fiber.

The reflectance of the first FP mirror is obtained due to the core discontinuity between the standard single-mode fiber and the suspended-core fiber. Considering the characteristics of the two fiber cores, a low reflectance is expected. The reflectance of the second FP mirror is due to the Fresnel reflection at the suspended-core fiber end. Fig. 2 shows the spectral response of the FP cavity with a four-hole suspended-core fiber before and after the splice with the hollow PCF. As can be observed, after splicing, the visibility and phase of the interferometer are changed. These variations are expected due to changes introduced by the electric arc at the end of the suspended-core fiber. Similar results were obtained for the case of the three-hole fiber. We should emphasize that the phase change is in major part due to impact of electric arc in length and refractive index of the fiber and it is not due to phase change in reflection.

The temperature characterization of the sensing heads was conducted at a constant strain. The sensors were placed in an oven where the temperature was set from room temperature up to $90{ }^{\circ} \mathrm{C}$, with an error smaller than $0.1^{\circ} \mathrm{C}$. Fig. 3 shows the

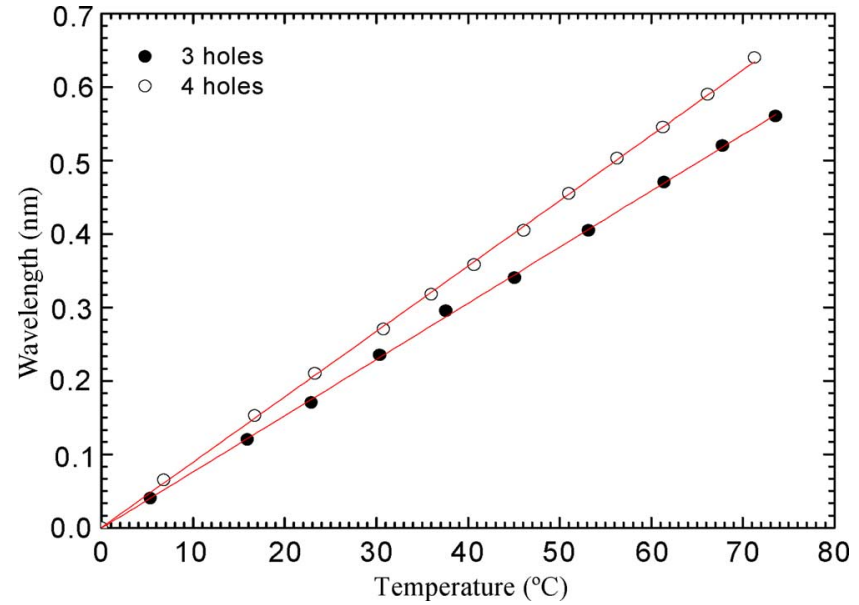

Fig. 3. Temperature response for the two FP cavities.

temperature responses of the two sensors. The sensitivity coefficients are 7.65 and $8.89 \mathrm{pm} /{ }^{\circ} \mathrm{C}$ for the fibers with three and four holes, respectively. The ratio between these two coefficients is $\approx 1.16$, which is approximately the ratio between the two cavity lengths (1.17), as it should be considering that the temperature sensitivity is essentially determined by the thermooptic coefficient of silica and, therefore, proportional to the cavity length (assuming that the two fibers have approximately the same modal characteristics [15]). The phase coefficients can be obtained using the relation $d \phi=\left(4 \pi n L / \lambda^{2}\right) d \lambda$, where $L$ is the cavity length, $n$ is the effective refractive index of the guided mode, and $\lambda$ is the operating wavelength (around $1550 \mathrm{~nm}$ ).

The strain responses of the sensors were also studied. As mentioned before, a length of hollow-core PCF was spliced to the end of the suspended-core fibers that permits strain measurements (see Fig. 1). The results are shown in Fig. 4. It is important to mention that the strain values indicated in this figure are average values. Due to the different silica areas in the cross sections of the standard single-mode fiber, the suspended-core fiber, and the hollow-core PCF, the strain is not constant along the sensing head [16]. A linear response to strain with slopes of 1.32 and $1.16 \mathrm{pm} / \mu \varepsilon$ for the cases of the suspended-core fibers with three and four holes, respectively, can be observed. Considering the geometry of the hollow-core PCF, the stress from this fiber is essentially applied to the cladding region of the suspended-core fibers, i.e., the stress is applied to the suspended core through the supporting walls. Looking at the inset photographs of Fig. 1, it turns out that the cladding cross-section area is smaller for the three-hole suspended-core fiber. Therefore, for a given stress applied to the fiber sensing head system, the elongation of the three-hole fiber segment is higher compared with the four-hole case, which explains the higher strain sensitivity of the FP sensing head relying on the three-hole suspended-core fiber. The temperature and strain sensitivity coefficients obtained for the studied sensing heads are grouped in Table I.

Due to the presence of holes in the fiber structure and the absence of doping in the core, it is expectable that these FP interferometers have larger strain sensitivity and smaller 


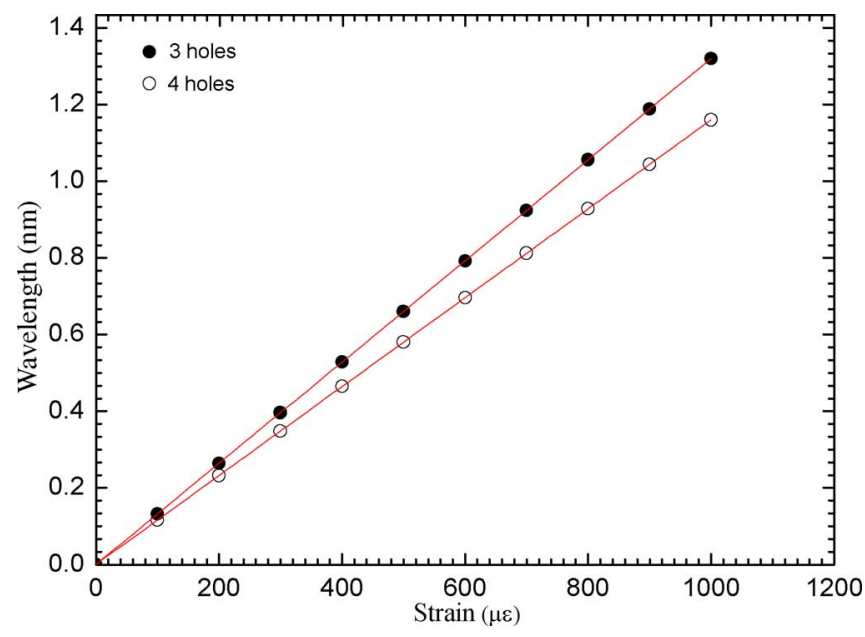

Fig. 4. Strain response for the two FP cavities.

TABLE I

STRAIN AND TEMPERATURE SENSITIVITY COEFFICIENTS

\begin{tabular}{lcc}
\hline \hline Fabry-Perot & $\begin{array}{c}\text { Strain } \\
(\mathrm{pm} / \mu \varepsilon)\end{array}$ & $\begin{array}{c}\text { Temperature } \\
\left(\mathrm{pm} /{ }^{\circ} \mathrm{C}\right)\end{array}$ \\
\hline Three-holes & 1.32 & 7.65 \\
Four-holes & 1.16 & 8.89 \\
\hline \hline & & \\
\hline \hline Fabry-Perot & $\begin{array}{c}\text { Strain } \\
\text { (normalized phase }\end{array}$ & $\begin{array}{c}\text { Temperature } \\
\text { (normalized phase } \\
\left.\text { sensitivity: } \mathrm{rad} / \mathrm{m} .{ }^{\circ} \mathrm{C}\right)\end{array}$ \\
\hline Three-holes & 10.4 & 67.8 \\
Four-holes & 8.5 & 67.6 \\
\hline \hline
\end{tabular}

temperature sensitivity when compared with FP cavities implemented in the standard single-mode fiber. Indeed, the results presented by Singh and Sirkis[17] confirm this statement in view of their reported sensitivities values of $2.1 \mathrm{rad} /(\mathrm{m} \cdot \mu \varepsilon)$ and $98.2 \mathrm{rad} /\left(\mathrm{m} \cdot{ }^{\circ} \mathrm{C}\right)$ for strain and temperature, respectively.

It is relevant to emphasize an important practical aspect of the proposed sensing structures. In view of the mismatch between the fundamental-mode effective indexes when it propagates in the suspended-core fibers, in standard single-mode fibers or in hollow-core PCFs, there is always a partially reflective surface at the splicing points of these fibers. Therefore, the fabrication of these miniaturized FP interferometers is relatively straightforward.

Although in this letter the strain and temperature measurement characteristics of these sensing structures were addressed, their applicability for measuring other quantities such as the external medium refractive index, hydrostatic pressure, or the dynamic pressure associated with acoustic waves is foreseeable.
Therefore, their study is proceeding, looking also for the development of operational software-based signal-processing techniques and the investigation of multiplexing layouts for multipoint measurement.

\section{CONCLUSION}

In this letter, two fiber FP interferometers based on suspended-core fibers with three and four holes was presented. The temperature and strain responses of these sensing structures were studied and compared with those found in related FP cavities built in standard single-mode fibers.

\section{REFERENCES}

[1] A. D. Kersey, D. A. Jackson, and M. Corke, "A simple fiber Fabry-Perot sensor," Opt. Commun., vol. 45, no. 2, pp. 71-74, Mar. 1983.

[2] C. E. Lee and H. F. Taylor, "Interferometric optical fiber sensors using internal mirrors," Electron. Lett., vol. 24, no. 4, pp. 193-194, Feb. 1988.

[3] F. Farahi, T. P. Newson, J. D. C. Jones, and D. A. Jackson, "Coherence multiplexing of remote fiber optic Fabry-Perot sensing system," Opt. Commun., vol. 65, no. 5, pp. 319-321, Mar. 1988.

[4] T. W. Kao and H. F. Taylor, "High-sensitivity intrinsic fiber-optic Fabry-Perot pressure sensor," Opt. Lett., vol. 21, no. 8, pp. 615-517, Apr. 1996.

[5] W. Tsai and C. Lin, "A novel structure for the intrinsic Fabry-Perot fiber-optic temperature sensor," J. Lightw. Technol., vol. 19, no. 5, pp. 682-686, May 2001.

[6] J. C. Knight, "Photonic crystal fibers," Nature, vol. 424, pp. 847-851, Aug. 2003

[7] O. Frazão, J. L. Santos, F. M. Araújo, and L. A. Ferreira, "Optical sensing with photonic crystal fiber," Laser Photon. Rev., vol. 2, no. 6, pp. 449-459, Dec. 2008

[8] C. E. Kerbage, B. J. Eggleton, P. S. Westbrook, and R. S. Windeler, "Experimental and scalar beam propagation analysis of an air-silica microstructure fiber," Opt. Express, vol. 7, pp. 13-122, 2000.

[9] B. J. Eggleton, C. Kerbage, P. S. Westbrook, R. S. Windeler, and A. Hale, "Microstructured optical fiber devices," Opt. Express, vol. 9, pp. 698-713, 2001

[10] Y. J. Rao, T. Zhu, X. C. Yang, and D. W. Duan, "In-line fiber-optic etalon formed by hollow-core photonic crystal fiber," Opt. Lett., vol. 32, no. 18, pp. 2662-2664, Sep. 2007.

[11] Z. L. Ran, Y. J. Rao, H. Y. Deng, and X. Liao, "Miniature in-line photonic crystal fiber etalon fabricated by $157 \mathrm{~nm}$ laser micromachining," Opt. Lett., vol. 32, no. 21, pp. 3071-3073, Nov. 2007.

[12] T. M. Monro, W. Belardi, K. Furusawa, J. C. Baggett, N. G. R. Broderick, and D. J. Richardson, "Sensing with microstructured optical fibers," Meas. Sci. Technol., vol. 12, pp. 854-858, Jan. 2001.

[13] A. S. Webb, F. Poletti, D. J. Richardson, and J. K. Sahu, "Suspendedcore holey fiber for evanescent-field sensing," Opt. Eng., vol. 46, no. 1, p. 010503-3, Jan. 2007

[14] O. Frazão, J. M. Baptista, J. L. Santos, J. Kobelke, and K. Schuster, "Strain and temperature characterization of a sensing head based on a suspended-core fiber in a Sagnac interferometer," Electron. Lett., vol. 44, no. 25, pp. 1455-1456, Dec. 2008.

[15] T. Martynkien, M. Szpulak, and W. Urbanczyk, "Modeling and measurement of temperature sensitivity in birefringent photonic crystal holey fibers," Appl. Opt., vol. 44, no. 36, pp. 7780-7788, Dec. 2005.

[16] O. Frazão, S. Silva, A. Guerreiro, J. L. Santos, L. A. Ferreira, and F. M. Araújo, "Strain sensitivity control of fiber Bragg gratings with fused tapers," Appl. Opt., vol. 46, no. 36, pp. 8578-8582, Dec. 2007.

[17] H. Singh and J. S. Sirkis, "Simultaneously measuring temperature and strain using optical fiber microcavities," J. Lightw. Technol., vol. 15 , no. 4, pp. 643-657, Apr. 1997. 